\title{
Influence of Crystal Habit and Particle Size Distribution on the Decomposition of a Solid. II. The Log-normal and the Super-hyperbolic Number Distributions
}

\author{
A R NE E N B ER G \\ Technical University of Denmark, Chemistry Department A, Building 207, \\ DK-2800 Lyngby, Denmark
}

\begin{abstract}
The topochemical kinetics has been derived for rectangular prismatic crystals with either log-normal or super-hyperbolic particle size distributions. It has been stressed that the influences of the crystal habit and particle size distribution on the decomposition kinetics can be treated as separate problems. Irrespective of the type of distribution, narrow distributions with identical dispersions are further shown to result in practically identical decomposition curves. Some of the results from the numeric calculations of the reduced decomposition curves (degree of reaction versus reduced time) have been tabulated for crystal cubes.
\end{abstract}

W hen a crystalline solid is heated or evacuated until a decomposition can be observed, the reaction rate will be determined by the following sequence of steps: heterogeneous nucleation, growth of nuclei, and diffusion of gaseous reaction products. When crystals are exposed to vacuum, the surface may be covered immediately by a product layer, whereafter the inwards movement of the reaction zone will be the rate determining step. Rate expressions can therefore simply be derived from the outer geometrical properties of the crystals.

The resulting topochemical kinetics have previously been described for single (or unisized) crystals of cubic, ${ }^{1}$ orthorhombic, ${ }^{2}$ and monoclinic ${ }^{3}$ symmetry and for size distributed crystals of cubic or spheric ${ }^{4}$ and orthorhombic ${ }^{5,6}$ symmetry. Trambouze and Imelik ${ }^{4}$ gave expressions for the decomposition of normal and logarithmic normal distributed spheres in terms of the mean particle diameter. Using the same diameter Delmon ${ }^{5}$ described the decomposition of parallelepipeds with different size distributions. Master curves were drawn for the degree of reaction versus a reduced time, which Delmon defined as the ratio between the actual time and the time corresponding to a degree of reaction of 0.9 . On the other hand Engberg ${ }^{6}$ considered

Acta Chem. Scand. 25 (1971) No. 10 
the decomposition of parallelepipeds with rectangular or narrow normal particle size distributions and defined the reduced time by means of the specific surface area. The master curves were thus transformed to curves with identical initial slopes. This greatly facilitates the evaluation of the kinetic parameters from experimental data, as the rate constants were obtained as the initial slopes of plots of $1-(1-\alpha)^{1 / 3}$ against time, where $\alpha$ is the degree of reaction. It was shown that a convexity of the experimental curves may be caused by a certain size distribution of uniform particles in the sample, while a concavity could be explained by a certain reaction anisotropy. It was further shown that narrow normal and rectangular size distributions may result in approximately the same kinetics.

In this paper the kinetics for particles with logarithmic normal and superhyperbolic size distributions are considered.

\section{THEORY}

For a sample of uniform prismatic crystals the volume $V(t)$ of unreacted material at the time $t$ can be written

$$
V(t)=\int_{2 k t}^{\infty}(a-2 k t)\left(l_{1} a-2 k t\right)\left(l_{2} a-2 k t\right) G(a) \mathrm{d} a
$$

$a$ being the smallest edge length of the crystal, $l_{1}$ and $l_{2}$ the elongations, $k$ the linear rate constant and $G(a)$ the frequency function determining the particle size distribution. Using this relation the degree of reaction $\alpha(t)$ is found from

$$
\alpha(t)=1-[V(t) / V(0)]
$$

In order to make the following expressions more general the particle size will be discussed in terms of the mode $a_{m}$ of the particle size distribution and the relative particle size $q$, so that $a$ may be replaced by $q a_{m}$. Finally, $2 k t / a_{m}$ is replaced by the reduced time $u$. The degree of reaction may then be written

$$
\alpha(t)=1-\frac{\int_{u}^{\infty}(q-u)\left(l_{1} q-u\right)\left(l_{2} q-u\right) G(q) d q}{\int_{0}^{\infty} l_{1} l_{2} G(q) q^{3} \mathrm{~d} q}
$$

which can be written out to give

$$
\begin{aligned}
\alpha(u)=1 & -\frac{\mathrm{I}_{3, u}}{\mathrm{I}_{3,0}}+\frac{\left(l_{1}+l_{2}+l_{1} l_{2}\right) \mathrm{I}_{2, u}}{l_{1} l_{2} \mathrm{I}_{3,0}} u-\frac{\left(1+l_{1}+l_{2}\right) \mathrm{I}_{1, u}}{l_{1} l_{2} \mathrm{I}_{3,0}} u^{2} \\
& +\frac{\mathrm{I}_{0, u}}{l_{1} l_{2} \mathrm{I}_{3,0}} u^{3}
\end{aligned}
$$

where $I_{n, r}$ denote the integrals

$$
\mathrm{I}_{n, r}=\int_{,}^{\infty} G(q) q^{n} \mathrm{~d} q
$$

which are dependent only on the relative particle size distribution. When these integrals are independent of time, which will be the case as long as 
none of the particles in the sample are totally decomposed, the degree of reaction is seen to be given as a polynomial of third degree in the reduced time. $\alpha(u)$ is now independent of the particle size (strictly speaking the mode of the particle size $a_{m}$ ). However, the initial slope of the reduced decomposition curve still depends on the shape of the particles in so far as the coefficient of $u$ in eqn. (4) contains the particle shape contribution to the specific surface area.

Many authors have analyzed the decomposition curves by plotting $1-(1-\alpha)^{1 / 3}$ against $t$ and they have obtained straight lines up to a degree of reaction of 0.4 or more, according to the equation

$$
1-(1-\alpha)^{1 / 3}=k_{3} t
$$

which is identical with the following equation

$$
\alpha(t)=3\left(k_{3} t\right)-3\left(k_{3} t\right)^{2}+\left(k_{3} t\right)^{3}
$$

Introducing the reduced time $x=k_{3} t$ we simply get

$$
\alpha(x)=3 x-3 x^{2}+x^{3}
$$

The latter expressions, however, should only be applicable for the decompositions of unisized spheres or cubes and otherwise eqn. (4) should be applied. However, the influence of particle shape and size distribution on the decomposition curve is most easily seen, when it is discussed in terms of deviations from the simple rate expression given in eqn. (8). For this purpose eqn. (4) is expressed in the $x$ variable using

to give

$$
x=\frac{\left(l_{1}+l_{2}+l_{1} l_{2}\right) \mathrm{I}_{2,0}}{3 l_{1} l_{2} \mathrm{I}_{3,0}} u
$$

$$
\alpha(x)=Q_{0}+3 Q_{1} x-\frac{9\left(1+l_{1}+l_{2}\right) l_{1} l_{2} Q_{2}}{\left(l_{1}+l_{2}+l_{1} l_{2}\right)^{2}} x^{2}+\frac{27 l_{1}^{2} l_{2}^{2} Q_{3}}{\left(l_{1}+l_{2}+l_{1} l_{2}\right)^{3}} x^{3}
$$

where the quantities

$$
Q_{0}=1-\frac{\mathrm{I}_{3, u}}{\mathrm{I}_{3,0}} ; Q_{1}=\frac{\mathrm{I}_{2, u}}{\mathrm{I}_{2,0}} ; Q_{2}=\frac{\mathrm{I}_{1, u} \mathrm{I}_{3,0}}{\mathrm{I}_{2,0}{ }^{2}} ; Q_{3}=\frac{\mathrm{I}_{0, u} \mathrm{I}_{3,0}{ }^{2}}{\mathrm{I}_{2,0}{ }^{3}}
$$

represent the contributions from the relative particle size distribution to the coefficients in the polynomial of $x$. Accordingly they will be named the distribution coefficients.

At the beginning of the decomposition, or strictly speaking in the time before that moment where the smallest particle has been totally decomposed, a change of the lower limit in the integrals $\mathrm{I}_{n, u}$ from $u$ to zero will have no influence of the result, meaning that $I_{n, u}=I_{n, 0}$. In this case the distribution coefficients take the values of

giving

$$
Q_{0}^{\circ}=0 ; Q_{1}^{\circ}=1 ; Q_{2}^{\circ}=\frac{\mathrm{I}_{1,0} \mathrm{I}_{3,0}}{\mathrm{I}_{2,0}{ }^{2}} ; Q_{3}^{\circ}=\frac{\mathrm{I}_{0,0} \mathrm{I}_{3,0}{ }^{2}}{\mathrm{I}_{2,0}{ }^{3}}
$$

$$
\alpha^{\circ}(x)=3 x-\frac{9\left(1+l_{1}+l_{2}\right) l_{1} l_{2} Q_{2}^{\circ}}{\left(l_{1}+l_{2}+l_{1} l_{2}\right)^{2}} x^{2}+\frac{27 l_{1}{ }^{2} l_{2}{ }^{2} Q_{3}^{\circ}}{\left(l_{1}+l_{2}+l_{1} l_{2}\right)^{3}} x^{3}
$$

Acta Chem. Scand. 25 (1971) No. 10 
where the superscripts denote this special case. However, this case does not necessarily need to be restricted to the beginning, since the equation may be satisfactory for the main part of the decomposition curve. For example when the particle size distribution is rectangular with a smallest particle size of half the mean, the conditions will be fulfilled for a degree of reaction up to at least 0.8. For still higher degrees of reaction eqn. (10) should be applied. The distribution coefficients will then be dependent on time giving a polynomial in $x$ of higher order than three.

When, at the beginning of this paper the reduced time $u$ was introduced instead of $2 k t / a_{m}, a_{m}$ was said to be the mode of the particle size distribution. We must emphasize that a continuous distribution with a frequence maximum was understood. In case of a discontinuous distribution $a_{m}$ will be used for the mean of the definition interval of particle size.

The reduced time $x$ can be defined by analogy with $u$ as $2 k t / a_{0}$, where $a_{0}$ denotes the cubic mean edge length, i.e. the edge length of a cube with the same specific surface area as the sample. The relation between $a_{0}$ and $a_{m}$ can be derived from eqn. (9) to give

$$
\frac{a_{0}}{a_{m}}=\frac{3 l_{1} l_{2} \mathrm{I}_{3,0}}{\left(l_{1}+l_{2}+l_{1} l_{2}\right) \mathrm{I}_{2,0}}
$$

The ratio is seen to be reduced to unity for a cube without a size distribution $\left(l_{1}=l_{2}=1\right.$ and $\left.I_{3,0}=I_{2,0}=1\right)$ in accordance with the definition.

In the following will be derived the distribution coefficients $Q_{2}{ }^{\circ}$ and $Q_{3}{ }^{\circ}$, the normalization factor $g$ and the ratio $a_{0} / a_{m}$ for some simple size distributions not dealt with in the previous paper. ${ }^{6}$

Log-normal distribution is considered as a model for the particle size distribution of undivided material. In this case the number distribution can be defined by the frequency function

$$
G(q)=g \exp \left[-\frac{3}{2}\left(\frac{\ln q}{s}\right)^{2}\right]
$$

where $g$ is the normalization factor and $s$ the distribution parameter. The latter is chosen cube root three times smaller than the normally defined standard deviation to bring the kinetic expressions in accordance with those relating to the discontinuous distributions. The problem is now to solve the integrals

$$
\mathrm{I}_{n, 0}=\int_{0}^{\infty} G(q) q^{n} \mathrm{~d} q=g \int_{0}^{\infty} q^{n} \exp \left[-\frac{3}{2}\left(\frac{\ln q}{s}\right)^{2}\right] \mathrm{d} q
$$

Replacement of the variable by

$$
y=\frac{(3)^{1 / 2} \ln q}{(2)^{1 / 2} s}
$$

results in

$$
\begin{aligned}
& \mathrm{I}_{n, 0}=\left(\frac{2}{3}\right)^{1 / 2} g s \int_{-\infty}^{\infty} \exp \left[-y^{2}+(n+1)(2 / 3)^{1 / 2} s y\right] \mathrm{d} y= \\
& (2 \pi / 3)^{1 / 2} g s \exp \left[\frac{1}{6}(n+1)^{2} s^{2}\right]
\end{aligned}
$$


which gives us

$$
Q_{2}^{\circ}=\exp \left(\frac{1}{3} s^{2}\right) ; \quad Q_{3}^{\circ}=\exp \left(s^{2}\right)
$$

Normalization of the frequency function

leads to

$$
\mathrm{I}_{0,0}=1=(2 \pi / 3)^{1 / 2} g s \exp \left(\frac{1}{6} s^{2}\right)
$$

$$
g=\frac{(3 / 2 \pi)^{1 / 2}}{s \exp \left(s^{2} / 6\right)}
$$

As the distribution is continuous, the smallest particles will already be decomposed at the very beginning. Thereafter the kinetic expression relating to the distribution coefficients given in eqn. (19) will result in more or less erroneous results. The accurate expression is obtained by solving the integrals

$$
\begin{gathered}
\mathrm{I}_{n, u}=\int_{u}^{\infty} G(q) q^{n} \mathrm{~d} q=\left(\frac{2}{3}\right)^{1 / 2} \int_{\left(\frac{3}{2}\right)^{1 / 2} \frac{\ln u}{8}}^{\infty} \exp \left[-y^{2}+(n+1)\left(\frac{2}{3}\right)^{\frac{1}{2}} s y\right] \mathrm{d} y
\end{gathered}
$$

Substitution of

yields

$$
z=y-\left(\frac{2}{3}\right)^{1 / 2}\left(\frac{n+1}{2}\right) s=\left(\frac{3}{2}\right)^{1 / 2}\left(\frac{\ln q}{s}-\frac{n+1}{3} s\right)
$$

$$
\begin{aligned}
\mathrm{I}_{n, u}=\left(\frac{2}{3}\right)^{1 / 2} g s \mathrm{e}^{\frac{1}{6}(n+1)^{2} s^{2}} & \int^{\infty} \exp \left[-z^{2}\right] \mathrm{d} z \\
& \left(\frac{3}{2}\right)^{1 / 2}\left(\frac{\ln u}{8}-\frac{n+1 s}{3}\right)
\end{aligned}
$$

Replacement of $u$ by $x$ according to eqns. (9) and (18) and introduction of the relative shape factor $f$ (in relation to that of the cube)

give that

$$
f=\frac{l_{1}+l_{2}+l_{1} l_{2}}{3 l_{1} l_{2}}
$$

$$
\mathrm{I}_{n, x}=\left(\frac{\pi}{6}\right)^{1 / 2} g s \mathrm{e}^{\frac{1}{6}(n+1)^{2} s^{2}}\left[1-\operatorname{erf}\left(\left(\frac{3}{2}\right)^{1 / 2}\left(\frac{\ln x}{s}+\frac{5-2 n}{6} s-\frac{\ln f}{s}\right)\right)\right]
$$

resulting in

$$
\begin{aligned}
& Q_{0}=\frac{1}{2}\left[1+\operatorname{erf}\left(\left(\frac{3}{2}\right)^{1 / 2}\left(\frac{\ln x}{s}-\frac{1}{6} s-\frac{\ln f}{s}\right)\right)\right] \\
& Q_{1}=\frac{1}{2}\left[1-\operatorname{erf}\left(\left(\frac{3}{2}\right)^{1 / 2}\left(\frac{\ln x}{s}+\frac{1}{6} s-\frac{\ln f}{s}\right)\right)\right] \\
& Q_{2}=\frac{1}{2}\left[1-\operatorname{erf}\left(\left(\frac{3}{2}\right)^{1 / 2}\left(\frac{\ln x}{s}+\frac{1}{2} s-\frac{\ln f}{s}\right)\right)\right] \mathrm{e}^{s^{2} / 3} \\
& Q_{3}=\frac{1}{2}\left[1-\operatorname{erf}\left(\left(\frac{3}{2}\right)^{1 / 2}\left(\frac{\ln x}{s}+\frac{5}{6} s-\frac{\ln f}{s}\right)\right)\right] \mathrm{e}^{s^{2}}
\end{aligned}
$$

Acta Chem. Scand. 25 (1971) No. 10 
When $x$ is small, the inner parentheses will be numerically large and negative, whereby the error functions tend towards -1 to give the size distribution coefficients already quoted in eqn. (19).

Superhyperbolic distributions are considered as models for the particle size distributions of sieve fractions. They are defined by the frequency functions

$$
G(q)=g q^{-m}
$$

within the range $1-s<q<1+s$ and are easily integrated to obtain the distribution coefficients. Because of the discontinuous distributions we must only remember that the integrals $I_{n, u}$ become

$$
\mathrm{I}_{n, u}=\int_{u}^{\infty} G(q) q^{n} \mathrm{~d} q=\begin{array}{ll}
g \int_{u}^{1+s} q^{n-m} \mathrm{~d} q & (1-s \leq u) \\
g \int_{1-s}^{1+s} q^{n-m} \mathrm{~d} q & (u \leq 1-s)
\end{array}
$$

For the long time region $(1-s<u)$ the distribution coefficients turn out to be

$$
Q_{0}=\frac{u^{2}-(1-s)^{2}}{4 s} ; Q_{1}=\frac{1+s-u}{2 s} ; Q_{2}=\frac{\ln [(1+s) / u]}{2 s} ; Q_{3}=\frac{1+s-u}{2 s(1+s) u}
$$

for the square-hyperbolic distribution, and

$$
\begin{aligned}
& Q_{0}=\frac{u-(1-s)}{2 s} ; Q_{1}=\frac{\ln [(1+s) / u]}{\ln [(1+s) /(1-s)]} ; Q_{2}=\frac{2 s(1+s-u)}{(1+s)\{\ln [(1+s) /(1-s)]\}^{2} u} \\
& Q_{3}=\frac{2 s^{2}\left((1+s)^{2}-u^{2}\right)}{(1+s)^{2}\{\ln [(1+s) /(1-s)]\}^{3} u^{2}}
\end{aligned}
$$

for the cube-hyperbolic distribution. The corresponding quantities for the short time region are shown in Table 2.

Table 1. Selected size distributions and their properties.

\begin{tabular}{|l|c|c|c|}
\hline \multicolumn{1}{|c|}{ Distribution } & $G(q)$ & $\begin{array}{c}\text { Definition } \\
\text { range }\end{array}$ & $\frac{a_{0}}{a_{m} f}=\frac{\mathrm{I}_{3,0}}{\mathrm{I}_{2,0}}$ \\
\hline Rectangular & $\frac{1}{2 s}$ & $1-s<q<1+s$ & $\frac{1+s^{2}}{1+s^{2} / 3}$ \\
Gaussian & $\frac{(3 / 2 \pi)^{1 / 2}}{s} \exp \left[-\frac{3}{2}\left(\frac{q-1}{s}\right)^{2}\right]$ & $0<q<\infty$ & $\frac{1+s^{2}}{1+s^{2} / 3}$ \\
Log-normal & $\frac{(3 / 2 \pi)^{1 / 2}}{8 \exp \left(s^{2} / 6\right)} \exp \left[-\frac{3}{2}\left(\frac{\ln q}{s}\right)^{2}\right]$ & $0<q<\infty$ & $\exp \left[\frac{7}{6} s^{2}\right]$ \\
Square-hyp. & $\frac{1-s^{2}}{2 s q^{2}}$ & $1-s<q<1+s$ & 1 \\
Cube-hyp. & $\frac{\left(1-s^{2}\right)^{2}}{2 s q^{3}}$ & $1-s<q<1+s$ & $\frac{2 s}{\ln \left(\frac{1+s}{1-s}\right)}$ \\
\hline
\end{tabular}


Table 2. Expansions of the size distribution coefficients.

\begin{tabular}{|c|c|}
\hline Distribution & $Q_{2}^{\circ}$ \\
\hline $\begin{array}{l}\text { Rectangular } \\
\text { or Gaussian }\end{array}$ & $\frac{1+s^{2}}{\left(1+s^{2} / 3\right)^{2}}=1+\frac{1}{3} s^{2}-\frac{1}{3} s^{4}+\frac{5}{27} s^{6}-\frac{7}{81} s^{8}+\cdots$ \\
\hline Log-normal & $\mathrm{es}^{2} / 3=1+\frac{1}{3} s^{2}+\frac{1}{6} s^{4}+\frac{1}{18} s^{6}+\frac{1}{72} s^{8}+\cdots$ \\
\hline Square-hyp. & $\frac{\ln \left(\frac{1+s}{1-s}\right)}{2 s}=1+\frac{1}{3} s^{2}+\frac{1}{5} s^{4}+\frac{1}{7} s^{6}+\frac{1}{9} s^{8}+\cdots$ \\
\hline Cube-hyp. & $\frac{1}{1-s^{2}}\left(\frac{2 s}{\ln \left(\frac{1+s}{1-s}\right)}\right)^{2}=1+\frac{1}{3} s^{2}+\frac{4}{15} s^{4}+\frac{44}{189} s^{6}+\frac{428}{2025} s^{8}+\cdots$ \\
\hline Distribution & $Q_{3}^{\circ}$ \\
\hline $\begin{array}{l}\text { Rectangular } \\
\text { or Gaussian }\end{array}$ & $\frac{1+s^{2}}{\left(1+s^{2} / 3\right)^{2}}=1+s^{2}-\frac{1}{4} s^{4}-\quad \frac{1}{9} s^{6}+$ \\
\hline Log-normal & $\mathrm{e}^{s^{2}}=1+s^{2}+\frac{1}{2} s^{4}+\frac{1}{6} s^{6}+\frac{1}{24} s^{8}+\cdots$ \\
\hline Square-hyp. & $\frac{1}{1-s^{2}}=1+s^{2}+\quad s^{4}+$ \\
\hline Cube-hyp. & $\frac{1}{\left(1-s^{2}\right)^{2}}\left(\frac{2 s}{\ln \left(\frac{1+s}{1-s}\right)}\right)^{3}=1+s^{2}+\frac{16}{15} s^{4}+\frac{1072}{945} s^{6}+\frac{16992}{14175} s^{8}+\cdots$ \\
\hline
\end{tabular}

\section{DISCUSSION}

The final results have been collected in Tables 1 and 2 . Which of the distributions will exert the greatest influence on the coefficients does not seem obvious, as the distribution coefficients take quite different forms for the different distributions. For this purpose the distribution coefficients have been expanded into the series quoted in Table 2. They show that it is not just the first term, but also the second, in the expansions, which is common for all distributions. This implies that the decomposition curves for narrow distributions (with $s$ less than 0.3 ) can be described with sufficient accuracy $(0.1 \%)$ up to a degree of reaction of at least 0.85 , by curves which are dependent only on the relative dispersion and not on the type of distribution. For broader distributions or longer times, however, the type of distribution may also play a role. It appears from Table 2 that the dependence of $Q_{2}{ }^{\circ}$ and $Q_{3}{ }^{\circ}$ on $s$ is smallest for the rectangular and greatest for the cube hyperbolic distribution. The convexity of the decomposition curves, with $s$ kept constant, will therefore increase in the falling order given in the table.

Acta Chem. Scand. 25 (1971) No. 10 
This can also be read from Table 3, where some of the results from the numeric calculations have been collected. The table shows the qualitative predictions to be fulfilled for reduced times less than about 0.6. For longer times the degree of reaction is increasing slower for the log-normal distribution because of the continuous nature of the distribution.

Table 3. Degree of reaction for size distributed cubes (or spheres, $l_{1}=l_{2}=1$ ) of different distribution types.

\begin{tabular}{cccccccccc}
\hline & & & & & & & \multicolumn{2}{c}{$s=0.9$} \\
$x$ & recta & log-n. & sq.hyp & cb.hyp. & recta & log-n. & sq.hyp. & cb.hyp. \\
\hline & & & & & & & & & \\
0.02 & 0.0587 & 0.0587 & 0.0587 & 0.0587 & 0.0587 & 0.0584 & 0.0581 & 0.0577 \\
0.04 & 0.1150 & 0.1149 & 0.1149 & 0.1148 & 0.1147 & 0.1139 & 0.1125 & 0.1110 \\
0.06 & 0.1688 & 0.1685 & 0.1684 & 0.1684 & 0.1682 & 0.1663 & 0.1635 & 0.1601 \\
0.08 & 0.2202 & 0.2198 & 0.2196 & 0.2195 & 0.2193 & 0.2160 & 0.2113 & 0.2055 \\
0.10 & 0.2693 & 0.2687 & 0.2684 & 0.2682 & 0.2679 & 0.2629 & 0.2562 & 0.2473 \\
0.12 & 0.3161 & 0.3153 & 0.3148 & 0.3146 & 0.3143 & 0.3073 & 0.2984 & 0.2860 \\
0.14 & 0.3607 & 0.3596 & 0.3591 & 0.3587 & 0.3584 & 0.3491 & 0.3382 & 0.3217 \\
0.16 & 0.4032 & 0.4018 & 0.4011 & 0.4006 & 0.4003 & 0.3886 & 0.3758 & 0.3549 \\
0.18 & 0.4436 & 0.4418 & 0.4410 & 0.4404 & 0.4402 & 0.4258 & 0.4113 & 0.3857 \\
0.20 & 0.4820 & 0.4798 & 0.4788 & 0.4782 & 0.4780 & 0.4608 & 0.4448 & 0.4146 \\
0.24 & 0.5529 & 0.5599 & 0.5486 & 0.5476 & 0.5479 & 0.5247 & 0.5066 & 0.4671 \\
0.28 & 0.6165 & 0.6125 & 0.6109 & 0.6096 & 0.6106 & 0.5811 & 0.5619 & 0.5138 \\
0.32 & 0.6731 & 0.6682 & 0.6662 & 0.6646 & 0.6664 & 0.6308 & 0.6116 & 0.5556 \\
0.36 & 0.7232 & 0.7173 & 0.7151 & 0.7130 & 0.7161 & 0.6745 & 0.6563 & 0.5934 \\
0.40 & 0.7674 & 0.7605 & 0.7580 & 0.7555 & 0.7600 & 0.7130 & 0.6963 & 0.6276 \\
0.44 & 0.8061 & 0.7981 & 0.7955 & 0.7926 & 0.7985 & 0.7468 & 0.7323 & 0.6587 \\
0.50 & 0.8548 & 0.8453 & 0.8427 & 0.8391 & 0.8475 & 0.7899 & 0.7794 & 0.7003 \\
0.60 & 0.9149 & 0.9032 & 0.9014 & 0.8965 & 0.9085 & 0.8454 & 0.8425 & 0.7585 \\
0.68 & 0.9476 & 0.9348 & 0.9343 & 0.9288 & 0.9424 & 0.8786 & 0.8814 & 0.7969 \\
0.76 & 0.9699 & 0.9568 & 0.9578 & 0.9521 & 0.9568 & 0.9042 & 0.9121 & 0.8295 \\
0.84 & 0.9841 & 0.9718 & 0.9741 & 0.9687 & 0.9813 & 0.9241 & 0.9361 & 0.8572 \\
0.92 & 0.9926 & 0.9818 & 0.9850 & 0.9803 & 0.9908 & 0.9397 & 0.9545 & 0.8807 \\
1.00 & 0.9971 & 0.9883 & 0.9919 & 0.9881 & 0.9961 & 0.9518 & 0.9684 & 0.9006 \\
\hline
\end{tabular}

As stressed earlier ${ }^{6}$ it could be remembered that the kinetic expressions derived here for an isotropic reaction may hold equally well for an anisotropic reaction. In the latter case the elongations to be used for calculation of the decomposition curve are different from the geometric ones determined by microscopy. It is supposed, however, that the particles are of uniform shape. This is an assumption which probably will never be strictly obeyed, but is believed to be considerably nearer the truth, than that the particles should be completely nonuniform. The decomposition should then have occurred as for a sample of unisized particles, the particle size distribution so being without any influence on the kinetics. For this reason the experimental decomposition curves may very well correspond to somewhat narrower particle size distributions than could be determined by microscopy. We may therefore conclude, that the deviations from the simplest kinetics for decomposition of a solid (unisized spheres or cubes) can be quantitatively explained by taking the 
particle shape and size distribution into consideration, though remembering that the parameters to be used in the explanation do not need to be identical with those determined from the outer geometrical properties of the particles.

In a later paper will be discussed some real particle size distributions and how to obtain the best approximations to the distribution models presented here.

Acknowledgement. Prof. N. Hofman-Bang and Prof. F. Woldbye are greatly appreciated for all facilities made available, and thanks are also expressed to the Northern Europe Computing Center in Lyngby for the access to the IBM 7094 computer.

\section{REFERENCES}

1. Roginski, S. and Schultz, E. Z. Phys. Chem. A 138 (1928) 21.

2. Hume, J. and Colvin, J. Proc. Roy. Soc. A 132 (1931) 548.

3. Eckhardt, R. C. and Flanagan, T. B. Trans. Faraday Soc. 60 (1964) 1289.

4. Trambouze, M. F. and Imelik, B. J. Chim. Phys. 57 (1960) 656.

5. Delmon, B. Rev. Inst. Fr. Pétrole 16 (1961) 1477.

6. Engberg, A. Acta Chem. Scand. 24 (1970) 931.

Received December 14, 1970. 